\title{
Carrier diffusion and higher order transversal modes in spectral dynamics of the semiconductor laser
}

Buus, Jens; Danielsen, Magnus

Published in:

I E E E Journal of Quantum Electronics

Link to article, DOI:

10.1109/JQE.1977.1069412

Publication date:

1977

Document Version

Publisher's PDF, also known as Version of record

Link back to DTU Orbit

Citation (APA):

Buus, J., \& Danielsen, M. (1977). Carrier diffusion and higher order transversal modes in spectral dynamics of the semiconductor laser. I E E E Journal of Quantum Electronics, 13(8), 669-674.

https://doi.org/10.1109/JQE.1977.1069412

\section{General rights}

Copyright and moral rights for the publications made accessible in the public portal are retained by the authors and/or other copyright owners and it is a condition of accessing publications that users recognise and abide by the legal requirements associated with these rights.

- Users may download and print one copy of any publication from the public portal for the purpose of private study or research.

- You may not further distribute the material or use it for any profit-making activity or commercial gain

- You may freely distribute the URL identifying the publication in the public portal 


\title{
Carrier Diffusion and Higher Order Transversal Modes in Spectral Dynamics of the Semiconductor Laser
}

\author{
JENS BUUS, STUDENT MEMBER, IEEE, AND MAGNUS DANIELSEN, MEMBER, IEEE
}

\begin{abstract}
The dynamic and spectral behavior of the semiconductor stripe laser has been investigated. For this purpose the rate equations have been generalized to include several longitudinal and transversal modes, spontaneous emission into the active modes, and position dependence of the electron density through a term describing the charge-carrier diffusion in the plane of the active layer. The parameters used for solving these equations are found by theoretical and experimental considerations. The results show a broadening of the spectrum together with a significant content of higher onder transversal modes along the junction plane.
\end{abstract}

\section{INTRODUCTION}

$\mathrm{T}$ HE understanding of the spectral dynamics of the semiconductor laser is of major importance for their applications in fiber optical communication systems. It is the purpose of this paper to work out a fundamental description of these properties based on numerical solution of a set of nonlinear multimode, position-dependent rate equations for the photon and the electron densities.

Earlier attempts to solve the rate equations by numerical methods have been based on a simple position-independent description involving the total photon density. In [1]-[3] a term for the spontaneous emission into the laser-active modes was not included. However, it has been shown that the magnitude of the spontaneous emission has a dominating influence on the ringings in the transient response [4]-[6]. For this situation, approximate analytical solutions have also been found [6]-[7]. Since the spectral width decreases with increasing current density, the total spontaneous emission into the lasing modes decreases with increasing current in the stationary state [8]. This can be taken into account in the theory of [4] - [7]. A more detailed description is obtained by using multimode rate equations. In [9] those equations with no position dependence were solved, taking the photon density for each mode as a variable. A position dependence of the spectral dynamics comes in through the diffusion of charge carriers in the active layer of the laser. The diffusion has been studied earlier for the stationary-state situation of the laser [10]-[12] .

In this paper, we have developed a more general model which describes the spectral and dynamic behavior during fast modulation dealing with both longitudinal and higher order transversal modes. The model consists of a set of multimode rate equations including spatial variations in both electron and photon densities where a special term accounts for the carrier diffusion. We have found that the existence of higher order modes is closely related to the carrier diffusion, and the results

Manuscript received November 22, 1976.

The authors are with the Electromagnetics Institute, Technical University of Denmark, Lyngby, Denmark. are in good qualitative agreement with experiments [13], [14]. In Section II we present our model in its general form; the laser parameters are discussed in Section III, and in Section IV the results which we have obtained are summarized.

\section{Spatial Variation of Electron AND Photon Densities}

In [1]-[9] all spatial variations are neglected. Since both the wavelength and the thickness of the active layer are much smaller than the diffusion length of the charge carrier, we neglect the spatial dependence along the stripe and perpendicular to the active layer. In the active layer where the stripe width is of the same order of magnitude as the diffusion length, we describe the diffusion of charge carrier by a diffusion term in the rate equation for the electron density.

Since the stripe width is much larger than the wavelength, one can expect to find higher order transversal modes. The photon energy depends on the longitudinal mode number $i$ but is almost independent of the transversal mode number $j$; hence the wavelength dependence of the gain can be described by the longitudinal mode number $i$ as $G_{i}$. The field distribution is assumed to be dependent only on the transverse mode number $j$. Using this, we find the rate equations

$$
\begin{aligned}
\frac{\partial n(x, t)}{\partial t}= & \frac{J(x, t)}{e d}-\frac{n(x, t)}{\tau_{s}}+D^{\prime} \frac{\partial^{2} n(x, t)}{\partial x^{2}} \\
& -\sum_{i, j} G_{i}(n(x, t))\left|E_{j}(x)\right|^{2} S_{i j} \\
\frac{d S_{i j}}{d t}= & \left(G_{i j}-\frac{1}{\tau_{p i j}}\right) S_{i j}+\frac{\beta_{i j}}{\tau_{s}} \frac{1}{2 s} \int_{-s}^{s} n(x, t) d x .
\end{aligned}
$$

The symbols are defined as follows: $n(x, t)$ and $J(x, t)$ are the electron and current densities at the position $x$ to the time $t$, $e$ the unit charge, $d$ the thickness of the active layer, $\tau_{s}$ the spontaneous carrier lifetime, $D^{\prime}$ the diffusion constant, $E_{j}(x)$ the normalized field distribution for a mode with the transversal mode number $j, S_{i j}$ the photon densities, $G_{i j}$, $\tau_{p i j}$, and $\beta_{i j}$ the mode gain, photon lifetime, and spontaneous-emission parameter for mode $(i, j)$, respectively, and $2 s$ the stripe width. The mode gain is given by

$$
G_{i j}=\frac{1}{2 s} \int_{-\infty}^{\infty} G_{i}(n(x))\left|E_{j}(x)\right|^{2} d x,
$$

where the normalization of the electric field

$$
\int_{-\infty}^{\infty}\left|E_{j}(x)\right|^{2} d x=2 s
$$


The field distribution $E_{j}(x)$ is, in principle, dependent on the gain and refractive index profiles [15], and hence on the electron density profile $n(x)$. This could be accounted for by a field equation. This, however, would complicate the solution of the equation drastically. For simplicity, we have used approximated fixed distributions.

Contrary to the multimode equation in [9], (1) is twodimensional since the position dependence is included, and it contains a second-order term.

In Section IV we describe some simplifications we have made in order to transform (1) to a set of one-dimensional first-order differential equations.

\section{Discussion of the Laser Parameters}

The simplest form of the rate equations is [6]

$$
\begin{aligned}
& \frac{d n}{d t}=\frac{J}{e d}-\frac{n}{\tau_{s}}-G S \\
& \frac{d S}{d t}=\left(G-\frac{1}{\tau_{p}}\right) S+\beta \frac{n}{\tau_{s}},
\end{aligned}
$$

where $S$ is the total photon density in the lasing modes, and $\beta$ is the total fraction of spontaneous light emitted into the lasing modes. The effective gain $G$ depends on the mean electron density $n$ only. Describing the gain by a Taylor expansion at the working point of the laser, a linear dependence on the electron density is obtained.

$$
G=\alpha\left(n-N_{0}\right) .
$$

Equations (5) and (6) have been treated analytically for the small-signal case [13], [16]-[18] and the solution is found to show a resonance behavior. It is then possible to find values for $\tau_{s}, \tau_{p}$, and $\beta$ by microwave modulation measurements.

From [13] we have $\tau_{s}=2 \mathrm{~ns}, \tau_{p}=23 \mathrm{ps}$, and $\beta=7.5 \cdot 10^{-3}$. The value of $\tau_{s}$, which also includes diffusion loss of electrons from the stripe region, is in good agreement with values currently used in the literature. Measurements of the turn on delay confirm this value. The values of $\tau_{p}$ and $\beta$ are surprisingly high. The lifetime of the photons, however, should be interpretated in conjunction with the gain. From (7) we obtain

$$
G-\frac{1}{\tau_{p}}=\alpha n-\frac{1}{\tau_{p}^{\prime}}
$$

with

$$
\tau_{p}^{\prime}=\frac{\tau_{p}}{1+\alpha N_{0} \tau_{p}} .
$$

From a numerical calculation of $G$ we have found $\alpha=0.62$. $10^{-12} \mathrm{~m}^{3} / \mathrm{s}, N_{0}=1.3 \cdot 10^{24} \mathrm{~m}^{-3}$, which gives $\tau_{p}^{\prime}=1.2 \mathrm{ps}$. We believe that the values of $\tau_{p}$ given in the literature, e.g., [16] and [18], are in fact $\tau_{p}^{\prime}$. Using (5) and (6), we find the threshold current density

$$
J_{t h}=\frac{1-\beta}{e d \alpha \tau_{p}^{\prime} \tau_{s}} .
$$

Hence if $J_{t h}$ is measured versus the mirror reflectivity one finds $\tau_{p}^{\prime}$ and not $\tau_{p}$. This is also the case if $\tau_{p}$ is found from resonance frequency and quantum efficiency measurements. In [13] we found $\tau_{p}$ on the basis of the damping parameter of the resonance curve.

The gain calculation is based on the assumptions of Lasher and Stern [19, formulas 9, 14, 15] (parabolic bands and no $k$ selection). The gain depends on the electron density through the fermi levels and on the photon energy as shown in Fig. 1.

The calculation of the gain for a given photon energy and electron density involves evaluation of the Fermi levels and integration over the energy axis. This procedure is too slow to use in a solution of the rate equations. Therefore we have developed a simplified description. As can be seen from Fig. 1, the spatial gain as a function of the photon energy $E$ for a given electron density can be approximated by a parabola

$$
g=g(n, E)=g_{\max }(n)-\left(E_{\max }(n)-E\right)^{2} P(n)^{2} .
$$

If $g_{\max }, E_{\max }$, and $P$ are expressed as parabolas in $n$, we obtain a simple formula for $g$. The 9 coefficients in this description are found from the exact gain curves. The temporal longitudinal gain is $G_{i}(n)=g\left(n, \lambda_{i}\right) \cdot c$, where $c$ is the light velocity in the laser.

The diffusion of charge carriers in the active layer involves both holes and electrons. Hence the effective diffusion constant $D^{\prime}$ is determined by combining the transport equations for holes and electrons in the active layer. After neglecting a nonlinear field term, we find for $D^{\prime}$

$$
D^{\prime}=\frac{\mu_{n} \mu_{p}}{\mu_{n}+\mu_{p}}\left(\frac{D_{n}}{\mu_{n}}+\frac{D_{p}}{\mu_{p}}\right)
$$

where $\mu_{n}$ and $\mu_{p}$ are mobilities, and $D_{n}$ and $D_{p}$ are diffusion constants for electrons and holes, respectively.

This formula was also used in [10]. When evaluating the parenthesis in (12) one must take into account that the conduction band is degenerated. Hence we use the generalized Einstein relations

$$
\begin{aligned}
& D_{p}=\alpha_{p} \mu_{p} \frac{k T}{e} \\
& D_{n}=\alpha_{n} \mu_{n} \frac{k T}{e},
\end{aligned}
$$

where $\alpha_{p}$ and $\alpha_{n}$ are functions of the hole and electron density, respectively. For electron densities in the range $10^{24}$ $\mathrm{m}^{-3}-2 \cdot 10^{24} \mathrm{~m}^{-3}$, we obtain from [20] $\alpha_{p} \approx 1, \alpha_{n} \approx 2$. Together with the fact $\mu_{n} \gg>\mu_{p}$ we find

$$
D^{\prime} \approx\left(\alpha_{n}+\alpha_{p}\right) \mu_{p} \frac{k T}{e} \approx 3 \mu_{p} \frac{k T}{e} .
$$

\section{RESULTS}

We have solved the position-dependent rate equations numerically by reformulation of the differential equation (1) into a set of difference equations with respect to the position. The subdivision of the active layer into zones with constant electron density can be made as fine as we want, but in order to save computer time we have used only five zones, two outside and three inside the stripe region. The calculations are outlined in Appendix I. This simple subdivision restricts the model to describing the fundamental and first-order trans- 


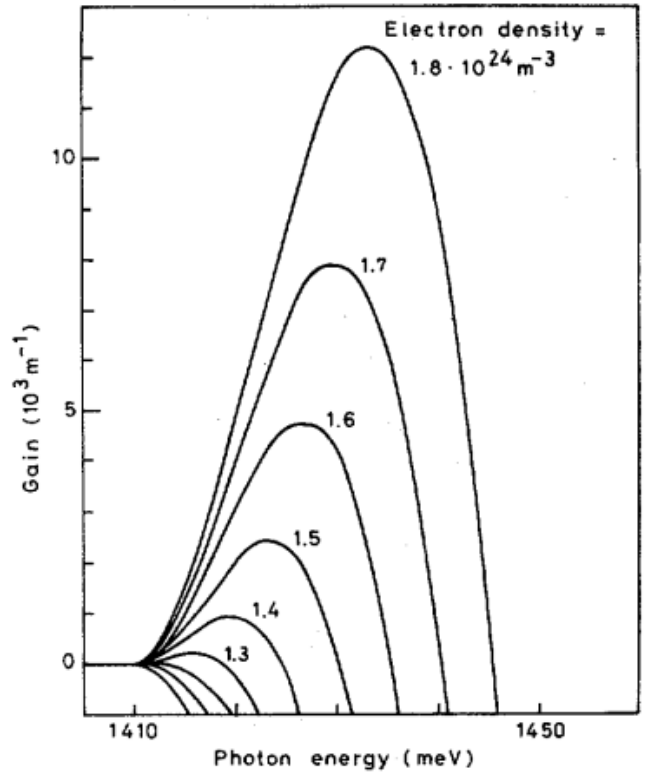

Fig. 1. Gain $g$ in a GaAs laser, intrinsic material, as a function of the photon energy with the electron density as a parameter. No $k$-selection rule.

versal modes in the plane of the active layer. The reason is that for each new pair of subregions (because of symmetry) we have an additional degree of freedom and therefore a new mode to describe the electromagnetic field.

The current is assumed to be confined in the active region neglecting current spreading [12]. The light is confined in the stripe region. Variations in the field distribution function $E_{j}(x)$ due to changes in the electron-density profile are neglected. Finally it should also be mentioned that thermal effects have been disregarded.

It has been proven that the grouping of the modes is an excellent method to save computer time in the numerical calculations. Taking two modes in each group, the grouping is done by a multiplication of both the mode spacing and the spontaneous-emission parameter per mode by two. Control calculations show that we obtain the same spectral distribution both for fundamental and first-order transversal modes irrespective of the number of modes per group. With a fundamental mode spacing of $4.25 \AA$ (corresponding to a laser length of $250 \mu \mathrm{m}$ ) and $\beta_{i j}=10^{-4}$, after grouping we get a mode spacing of $8.5 \AA$ and $\beta_{i j}=2.10^{-4}$.

For the carrier lifetime we use a value of 3 ns. This is in agreement with the measured effective lifetime of $2 \mathrm{~ns}$, since in (1), the diffusion is accounted for explicitly. Using $\mu_{p}=$ $0.017 \mathrm{~m}^{2} / \mathrm{V} \cdot \mathrm{s}$, [21], we find $D^{\prime}=1.3 \cdot 10^{-3} \mathrm{~m}^{2} / \mathrm{s}$ (at $300 \mathrm{~K}$ ). From this we have the effective diffusion length $L^{\prime}=\sqrt{D^{\prime} \tau_{s}}=$ $1.97 \mu \mathrm{m}$. For the stripe width $2 s=10 \mu \mathrm{m}$, the zone widths (see Fig 8) become $a=3.33 \mu \mathrm{m}, b=2.2 \mu \mathrm{m}$.

We measure current densities in units of the threshold current density $J_{t h}$. This quantity is defined in Appendix II.

In Fig. 2 we show the calculated ratio between the total photon density in first-order modes and the total photon density in the fundamental modes as a function of the current density. For current densities from $J \approx 1.05 J_{\text {th }}$ to $J \approx 1.2$ $J_{t h}$, only fundamental modes are present. Below threshold,

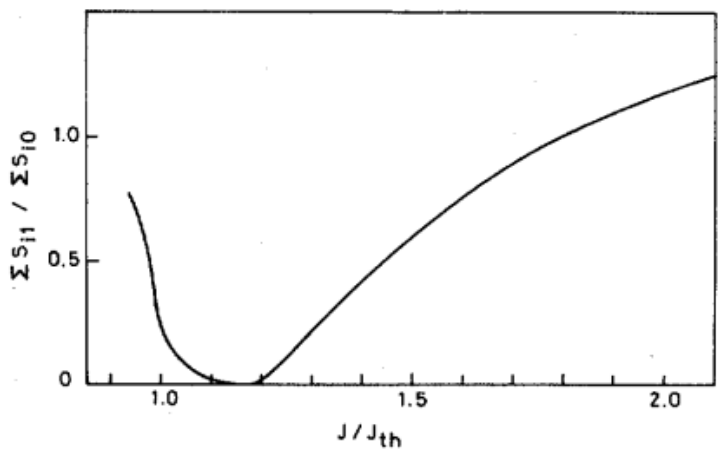

Fig. 2. Ratio of total photon density in first-order transversal modes to total photon density in the fundamental modes in static state as a function of current density.

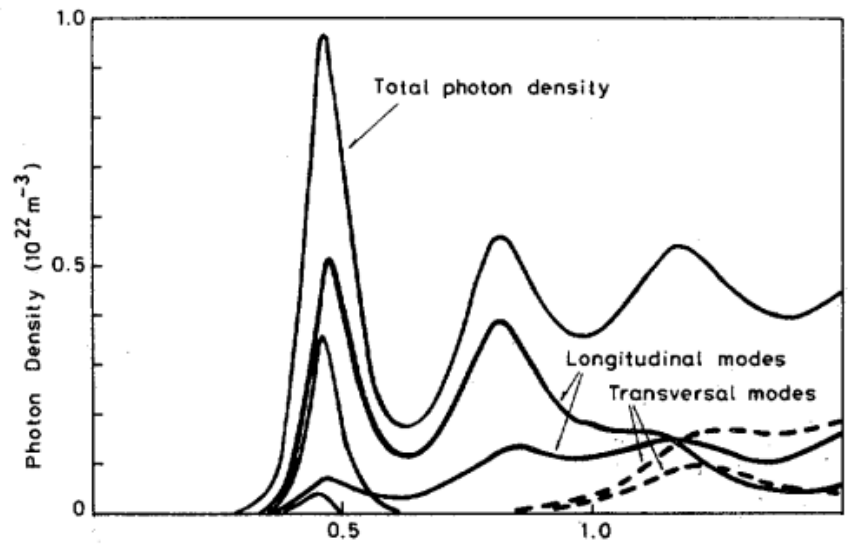

Fig. 3. Time evolution of total photon density, and photon densities in fundamental and first-order transversal modes in several longitudinal mode groups with two modes in each. The mode spacing was $4.25 \AA$, the bias current $0.95 \times$ threshold current, and current step $0.65 \times$ threshold current.

spontaneous emission dominates and many types of modes are seen. A high photon density in the fundamental modes will give strong stimulated emission in the central part of the active region (Zone 1). This will decrease the electron density in this zone. Hence the gain profile becomes flatter, giving a higher gain for first-order modes, and these modes will dominate for $J \geqslant 1.8 J_{t h}$.

We have solved the rate equations for the case of a step current

$$
\begin{array}{ll}
J=J_{B}, & t \leqslant 0 \\
J=J_{B}+J_{S}, & t>0 .
\end{array}
$$

In the following, we shall discuss the results for $J_{B}=0.95$ $J_{t h}, J_{S}=0.65 J_{t h}$. Figs. 3 and 4 show the photon and electron densities. We note that the total photon density exhibits the well-known transients. A qualitative discussion of this phenomenon is given in [6], [7].

In Fig. 5 we show the ratio between the modes $(n, 0)$ and $(n$, 1 ) as a function of time. The oscillation between the fundamental and the first order is in qualitative agreement with experimental results [13], [14].

The physical mechanism behind this curve is as follows: at the prebias current level below threshold the spontaneous 


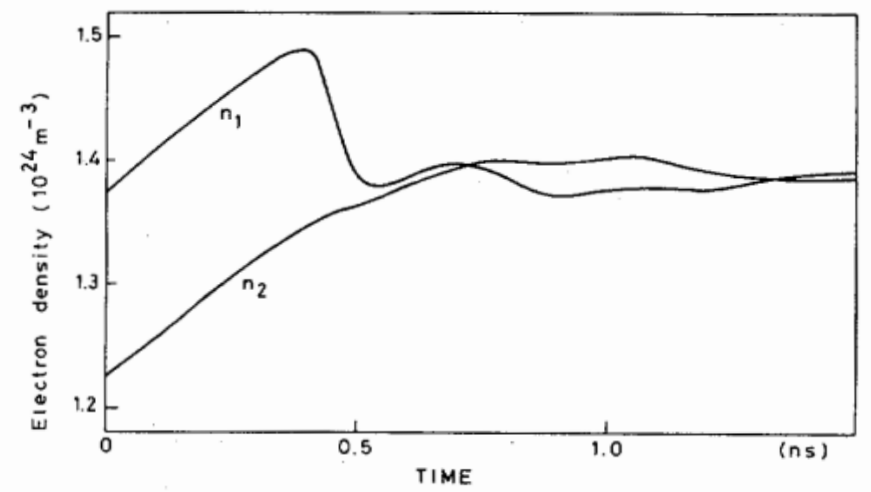

Fig. 4. Time evolution of electron density in central and border zones of stripe. Conditions were the same as in Fig. 3.

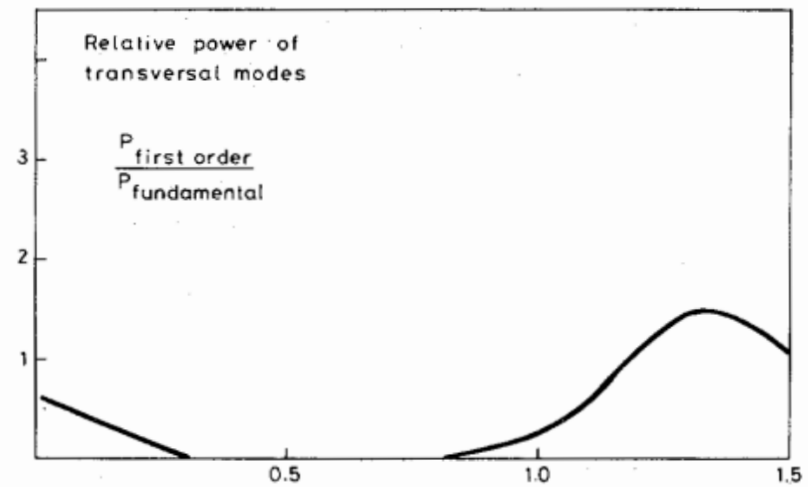

Fig. 5. Time evolution of the ratio of power in the first-order transversal mode to the fundamental mode corresponding to the longitudinal mode dominating in the static state. Conditions were the same as in Fig. 3.

emission dominates, thus exciting all modes, including the transverse modes, almost equally in power. When the current pulse is applied, the electron density and hence the gain in the three central zones will grow, amplifying both fundamental and first-order modes. The fundamental modes, however, have the greatest mode gain-due to the outdiffusing of electrons to the two passive regions outside the stripe-and will reach threshold first, causing the relaxation spikes to appear. The laser action in the fundamental modes will remove electrons from the central regions (spatial hole burning) and can give a spatial minimum of the electron density, while the two border zones in the stripe will be pumped further, increasing the first-order mode intensities. After the second spike, first-order modes may even dominate before a steady state is reached.

From the calculations we also find the spectral width as a function of time (Fig. 6). This result is in good agreement with experiments from [13], [14]. In [22] a slower variation is found. Since the photon energy at which the gain has its maximum value depends on the electron density, the wavelength of maximum photon density will depend on the time (Fig. 7).

The difference between the maximum wavelength for the fundamental and the first-order modes will depend on differences in the photon lifetimes; in this case the same photon lifetime was used.

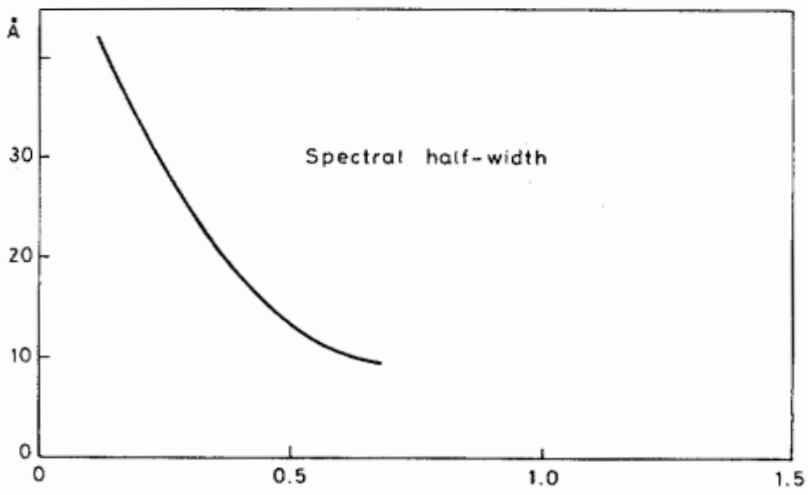

Fig. 6. Time evolution of spectral width with the same conditions as in Fig. 3.

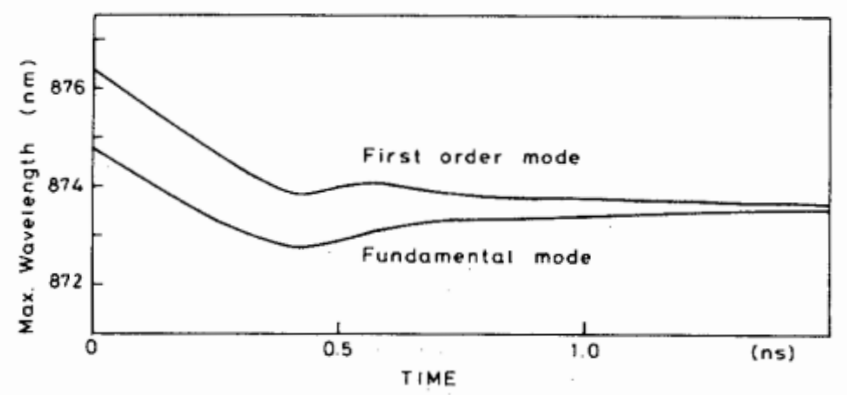

Fig. 7. Time evolution of the wavelengths for the fundamental and first-order transversal modes with maximum photon contents. The conditions were the same as in Fig. 3.

For the time interval $T$ between the first two spikes, the following relation is found to be in agreement with the simple rate equations (5), (6) [6], [7].

$$
T \approx\left(\alpha J_{S}\right)^{-1 / 2},
$$

where $\alpha$ is given by (7). The calculated value of $T$ is also in agreement with experimental results [13]-[14]. From the gain curves we find that $\alpha$ decreases with a decreasing value of the critical electron density, and since $n_{c}$ decreases with increasing value of the photon lifetime, we see that $T$ increases with increasing $\tau_{p}$ and we take the agreement between calculated and observed values of $T$ as a verification of the large value of $\tau_{p}$.

\section{CONCLUSION}

With the model described in this paper we are able to account for both spectral behavior and the existence of firstorder modes in the transients of a GaAlAs laser. The results are in qualitative agreement with experiments.

The spectral width of the laser will be of practical interest for system considerations, only in the case of fibers having carefully controlled index profiles. On the other hand changes from fundamental to first-order modes might have a severe effect on the coupling to fibers with a small normalized frequency [23]. The dynamic behavior is of interest in digital communication systems with directly modulated lasers since the ringing transients set a limit on the maximum modulation speed [7]. 


\section{APPENDIX I}

The active region of a semiconductur laser has the typical dimensions of the order of magnitude shown in Fig. 8, 2s being the stripe width. In order to describe the transversal variations the active layer is divided into five zones. Due to the symmetry it is only necessary to consider three zones: 1 , $2-2^{\prime}$, and $3-3^{\prime}$. The electron density is approximated by

$$
n(x)=\left\{\begin{array}{lll}
n_{1}, & \text { for } & |x| \leqslant \frac{s}{3} \\
n_{2}, & \text { for } & \frac{s}{3}<|x| \leqslant s \\
n_{3}, & \text { for } & s<|x| \leqslant b+s .
\end{array}\right.
$$

Since the precise variation of the light intensity is of minor importance, the following trigonometrical description is used:

$$
\begin{aligned}
& \left|E_{0}\right|= \begin{cases}\sqrt{2} & \cos \left(\frac{\pi x}{2 s}\right)|x| \leqslant s \\
0 & |x|>s\end{cases} \\
& \left|E_{1}\right|= \begin{cases}\sqrt{2} & \sin \left(\frac{\pi x}{s}\right)|x| \leqslant s \\
0 & |x|>s\end{cases}
\end{aligned}
$$

(only fundamental and first-order modes are considered).

The mode gain becomes

$$
\begin{aligned}
G_{i j} & =\frac{1}{2 s} \int_{-\infty}^{\infty} G_{i}(n(x))\left|E_{j}(x)\right|^{2} d x \\
& =\frac{1}{2 s} G_{i}\left(n_{1}\right) \int_{-(s / 3)}^{s / 3}\left|E_{j}\right|^{2} d x+\frac{1}{s} G_{i}\left(n_{2}\right) \int_{s / 3}^{s}\left|E_{j}\right|^{2} d x,
\end{aligned}
$$

using

$$
\begin{gathered}
\int_{-(s / 3)}^{s / 3}\left|E_{j}\right|^{2} d x= \begin{cases}\frac{2}{3} s\left(1+\frac{3 \sqrt{3}}{2 \pi}\right) \sim 1.2 s, & \text { for } j=0 \\
\frac{2}{3} s\left(1-\frac{3 \sqrt{3}}{4 \pi}\right) \sim 0.4 s, & \text { for } j=1\end{cases} \\
\int_{s / 3}^{s}\left|E_{j}\right|^{2} d x= \begin{cases}\frac{2}{3} s\left(1-\frac{3 \sqrt{3}}{4 \pi}\right) \sim 0.4 s, & \text { for } j=0 \\
\frac{2}{3} s\left(1+\frac{3 \sqrt{3}}{8 \pi}\right) \sim 0.8 s, & \text { for } j=1\end{cases}
\end{gathered}
$$

We define the gain for fundamental and first order modes by

$$
\begin{aligned}
\dot{G}_{i 0} & =0.6 G_{i}\left(n_{1}\right)+0.4 G_{i}\left(n_{2}\right) \\
G_{i 1} & =0.2 G_{i}\left(n_{1}\right)+0.8 G_{i}\left(n_{2}\right) .
\end{aligned}
$$

The average electron density in the stripe region is

$$
\frac{1}{2 s} \int_{-s}^{s} n(x) d x=\frac{n_{1}+2 n_{2}}{3} \text {. }
$$

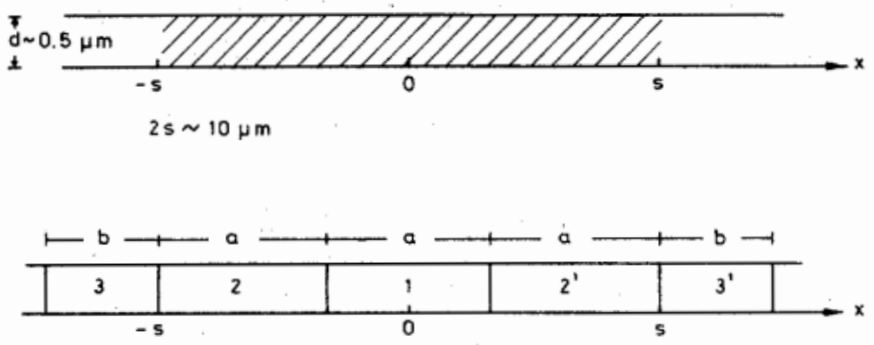

Fig. 8. Dimensions of the active region of laser, and the subdivision in zones used in computer calculations.

Instead of the photon lifetimes for fundamental and firstorder modes $\tau_{p 0}$ and $\tau_{p 1}$ we define the photon lifetimes for zones 1 and $2-2^{\prime}$ by

$$
\begin{aligned}
\tau_{p}^{*} & =\left(2 \tau_{p 0}^{-1}-\tau_{p 1}^{-1}\right)^{-1} \\
\tau_{p}^{* *} & =\left(-\frac{1}{2} \tau_{p 0}^{-1}+\frac{3}{2} \tau_{p 1}^{-1}\right)^{-1} .
\end{aligned}
$$

We can write the equations for the photon densities:

$$
\begin{aligned}
\frac{d S_{i 0}}{d t}= & \left(0.6\left(G_{i}\left(n_{1}\right)-\tau_{p}^{*-1}\right)+0.4\left(G_{i}\left(n_{2}\right)-\tau_{p}^{* *-1}\right)\right) S_{i 0} \\
& +\frac{\beta_{i}}{\tau_{s}} \frac{n_{1}+2 n_{2}}{3} \\
\frac{d S_{i 1}}{d t}= & \left(0.2\left(G_{i}\left(n_{1}\right)-\tau_{p}^{*-1}\right)+0.8\left(G_{i}\left(n_{2}\right)-\tau_{p}^{* *-1}\right)\right) \dot{S}_{i 1} \\
& +\frac{\beta_{i}}{\tau_{s}} \frac{n_{1}+2 n_{2}}{3} .
\end{aligned}
$$

The electron flux $\Phi$ due to diffusion is given by Fick's law

$$
\Phi=D^{\prime} \frac{d n}{d x}
$$

$D^{\prime}$ being the diffusion constant. The density change per unit time in the volume $V$ due to diffusion through an area $A$ is

$$
\left.\frac{d n}{d t}\right|_{\mathrm{DIF}}=\Phi \frac{A}{V} \text {. }
$$

As an example we consider diffusion from zone $2-2^{\prime}$ to zone 1 ; we find

$$
\begin{aligned}
\frac{d n}{d x} & \approx \frac{n_{2}-n_{1}}{a} \\
A & =2 L d \quad(L \text { is the laser length }) \\
V & =a L d \\
\left.\frac{d n}{d t}\right|_{\text {DIF }} & =D^{\prime} \frac{2}{a^{2}}\left(n_{2}-n_{1}\right) .
\end{aligned}
$$

With this procedure (1) can be written as three equations:

$$
\begin{aligned}
\frac{d n_{1}}{d t}= & \frac{J}{e d}-\frac{n_{1}}{\tau_{s}}+D^{\prime} \frac{2}{a^{2}}\left(n_{2}-n_{1}\right)-1.8 \sum_{i} G_{i}\left(n_{1}\right) S_{i 0} \\
& -0.6 \sum_{i} G_{i}\left(n_{1}\right) S_{i 1}
\end{aligned}
$$




$$
\begin{aligned}
\frac{d n_{2}}{d t}= & \frac{J}{e d}-\frac{n_{2}}{\tau_{s}}+D^{\prime} \frac{1}{a^{2}}\left(n_{1}-n_{2}\right)+D^{\prime} \frac{2}{(a+b) a}\left(n_{3}-n_{2}\right) \\
& -0.6 \sum G_{i}\left(n_{2}\right) S_{i 0}-1.2 \sum G_{i}\left(n_{2}\right) S_{i 1} \\
\frac{d n_{3}}{d t}= & \frac{n_{3}}{\tau_{s}}+D^{\prime} \frac{2}{(a+b) b}\left(n_{2}-n_{3}\right) .
\end{aligned}
$$

The width of the passive zone $3-3^{\prime}$ is found by using the condition that the ratio $n_{3} / n_{2}$ found from (A14)-(A16) should have the same value as the corresponding ratio for the average electron densities found from (1), when stimulated emission is neglected. We find typically

$$
b \sim L^{\prime}=\sqrt{D^{\prime} \tau_{s}},
$$

$L^{\prime}$ being the effective diffusion length.

\section{APPENDIX II}

In order to define a threshold current density for fundamental modes, we first define the critical electron density $n_{c}$

$$
G\left(n_{c}\right)=\frac{1}{\tau_{p 0}}=\frac{0.6}{\tau_{p}{ }^{*}}+\frac{0.4}{\tau_{p}{ }^{* *}} \text {. }
$$

Assuming that $G$ is linear in $n$, we obtain, from $0.6 G\left(n_{1}\right)+$ $0.4 G\left(n_{2}\right)=G\left(n_{c}\right)$,

$$
0.6 n_{1}+0.4 n_{2}=n_{c} \text {. }
$$

For a given current density we find $n_{1}$ and $n_{2}$ from (A14)(A16) by setting $d / d t=0$, and neglecting stimulated emission. The current density for which (A19) is satisfied is then defined as the threshold current density.

\section{REFERENCES}

[1] T. Ikegami, K. Kobayashi, and Y. Suematsu, "Transient behaviour of semiconductor injection lasers," Electron. Commun. Jap., vol. 53B, pp. 82-89, 1970.

[2] M. J. Adams, "Rate equations and transient phenomena in semiconductor lasers," Opt. Electron., vol. 5, pp. 201-215, 1973.

[3] W. Harth, "Large signal direct modulation of injection lasers," Electron Lett., vol. 9, pp. 532-533, 1973.

[4] R. Dunsmuir, "Theory of relaxation oscillations in optical lasers," J. Electron. Contr., vol. 10, pp. 453-458, 1961.
[5] D. Röss, "Einfuss der spontanen Emission auf das Einschwingverhalten von Lasern," $Z$. Naturforsch., vol. 19a, pp. 1169$1177,1964$.

[6] P. M. Boers, M. Danielsen, and M. T. Vlaardingerbroek, "Dynamic behaviour of semiconductor lasers," Electron. Lett., vol. 11, pp. 206-208, 1975.

[7] M. Danielsen, "A theoretical analysis of gigabit/second pulse code modulation of semiconductor lasers," IEEE J. Quantum Electron., vol. QE-12, pp. 657-660, Nov. 1976.

[8] R. Salathe, C. Voumard, and H. Weber, "Rate equation approach for diode lasers," Opt. Electron., vol. 6, pp. 451-456, 1974.

[9] H. Hildebrand and P. Russer, "Large signal PCM behaviour of injection lasers with coherent irradiation into one of the oscillating modes," Electron Lett., vol. 11, pp. 372-374, 1975.

[10] H. Statz, C. L. Tang, and J. M. Lavine, "Spectral output of semiconductor lasers," J. Appl. Phys., vol. 35, pp. 2581-2585, 1964.

[11] B. W. Hakki, "Carrier and gain spatial profiles in GaAs stripe geometry lasers," J. Appl. Phys., vol. 44, pp. 5021-5029, 1973.

[12] - "GaAs double heterostructure lasing behaviour along the junction plane," J. Appl. Phys., vol. 46, pp. 292-302, 1975.

[13] J. Buus, M. Danielsen, P. Jeppesen, F. Mengel, H. Moeskjær, and V. Ostoich, "On the dynamical and spectral behaviour of GaAs lasers modulated with subnanosecond pulses," in Proc. 2nd European Conf. Optical Fibre Commun., Paris, France, Sept. 1976, pp. 231-279.

[14] F. Mengel and V. Ostoich, "Dynamics of longitudinal and transversal modes along the junction plane in GaAlAs stripe lasers," IEEE J. Quantum Electron., vol. QE-13, pp. 359-361, May 1977.

[15] F. R. Nash, "Mode guidance parallel to the junction plane of double-heterostructure GaAs lasers," J. Appl. Phys., vol. 44, pp. 4696-4706, 1973.

[16] T. Ikegami and Y. Suematsu, "Direct modulation of semiconductor junction laser," Electron. Commun. Jap., vol. 51B, pp. 51-58, 1968.

[17] T. L. Paoli and J. E. Ripper, "Direct modulation of semiconductor junction laser," Proc. IEEE, vol. 58, pp. 1457-1465, 1970 .

[18] T. Ikegami and Y. Suematsu, "Carrier lifetime measurement of a junction laser using direct modulation," IEEE J. Quantum Electron., vol. QE-4, pp. 148-151, Apr. 1968.

[19] G. Lasher and F. Stern, "Spontaneous and stimulated recombination radiation in semiconductors," Phys. Rev., vol. 133, pp. $553-563,1964$.

[20] K. A. Shore and M. J. Adams, "The effects of carrier degeneracy on transport properties of the double heterostructure injection laser," Appl. Phys., vol. 9, pp. 161-164, 1976.

[21] S. M. Sze, Physics of Semiconductor Devices. New York: Wiley, 1969, p. 40.

[22] T. Ikegami, "Spectrum broadening and tailing effect in directmodulated injection lasers," in Proc. 1st European Conf. Optical Fibre Commun., London, England, 1975, p. 111.

[23] D. Gloge, "Dispersion in weakly guiding fibers," Appl. Opt., vol. 10, pp. 2442-2445, 1971. 